\title{
iCharibot: Design and Field Trials of a Fundraising Robot
}

Miguel Sarabia, Tuan Le Mau, Harold Soh, Shuto Naruse, Crispian Poon, Zhitian Liao, Kuen Cherng Tan, Zi Jian Lai, and Yiannis Demiris

Personal Robotics Lab Department of Electrical and Electronic Engineering Imperial College London, United Kingdom

\{miguel.sarabia, y.demiris\}@imperial.ac.uk

\begin{abstract}
In this work, we address the problem of increasing charitable donations through a novel, engaging fundraising robot: the Imperial Charity Robot (iCharibot). To better understand how to engage passersby, we conducted a field trial in outdoor locations at a busy area in London, spread across 9 sessions of 40 minutes each. During our experiments, iCharibot attracted 679 people and engaged with 386 individuals. Our results show that interactivity led to longer user engagement with the robot. Our data further suggests both saliency and interactivity led to an increase in the total donation amount. These findings should prove useful for future design of robotic fundraisers in particular and for social robots in general.
\end{abstract}

\section{Introduction}

In 2011, an estimated $£ 11$ bn were raised for charities in the UK, with almost 6 in every 10 adults contributing to charitable causes [5]. That said, charities still face challenges collecting sufficient funds in our current harsh economic climate. In fact, charitable giving fell $11 \%$ in 2009 back to 2007 levels. In the 2011 UK Giving Report it was highlighted that "the operating environment for the sector remains tough ... and a number of organisations are having to scale back or indeed cease activity in light of financial difficulties" [5].

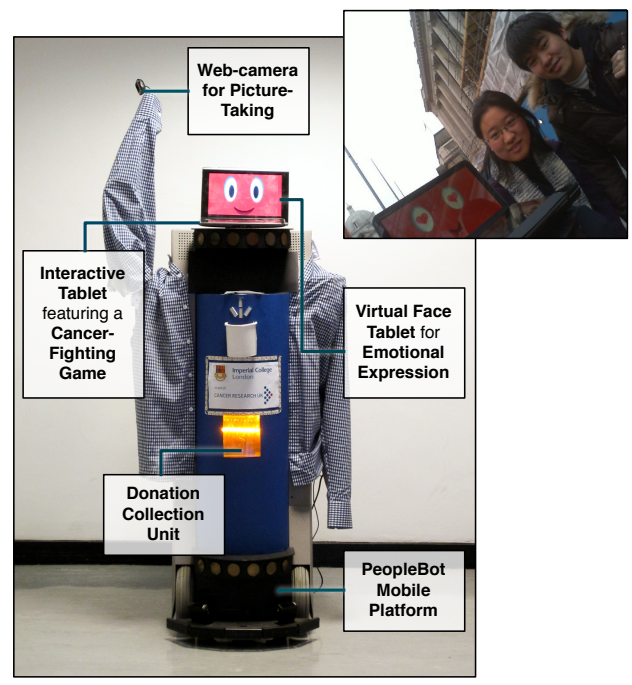

Fig. 1. Picture of iCharibot highlighting the main components of the robot, and two participants who engaged with the it ${ }^{1}$.

\footnotetext{
${ }^{1} \mathrm{~A}$ video of the system is available at: http://imperial.ac.uk/PersonalRobotics.
} 
In this work, we consider raising funds for charitable causes with an engaging donation-collection robot we call the Imperial Charity Robot (iCharibot), shown in Fig. 1. iCharibot can express a range of facial expressions and has several abilities useful for social interaction; for example, waving hello and and offering passers-by to play a tablet game.

From a human-robot interaction (HRI) research perspective, we are interested in understanding the interaction mechanisms that would attract people to approach the robot and, hopefully, persuade them to donate. In other words, how should an engaging charity robot act and behave? In an effort to answer this question, we performed field trials spread across 9 sessions of 40 minutes each in three real-world locations in the South Kensington area of London; a busy locale, home to three museums, a concert hall and our university. During the 9 sessions, 386 people engaged with the robot and a total of $£ 116.77$ was raised. All funds collected by iCharibot were donated to Imperial Cancer Research, a subsidiary of Cancer Research UK.

Although there have been many robots interacting with the public in unconstrained environments $[3,13]$; the goal of such studies has traditionally been to verify whether a system is functional rather than to investigate robotic design principles. Further, iCharibot is uncommon amongst fundraising robots $[7,8]$ in that it is the only one to have been deployed in a busy urban street.

This paper describes iCharibot's design and the results of our field trials. Specifically, we find that a more sophisticated behaviour (this concept is defined in Sect. 3.3) leads to greater user engagement duration and suggests a greater amount of donations.

\section{Background}

To the best of our knowledge, our work is preceded by only two other cases of fundraising robots. The first was Dona [7], which unlike iCharibot, was designed to be cat-like. As such, Dona had a very small foot-print and was designed to roam around its environment. From the point of view of interaction Dona bowed repeatedly when it encountered a person or an object. In contrast, iCharibot has a different roster of available actions, like waving its arms or reproducing synthesised speech.

The second fundraising robot was RoboBeggar, which took the appearance of a traditional Finnish begging statue [8]. RoboBeggar was similar in size to iCharibot and also had a touch screen for interaction but used a bank-card reader instead of a coinbox. The robot collected donations on behalf the Cancer Association of South-West Finland for two weeks at a shopping centre in Turku. A key difference between that experiment and ours was that RoboBeggar shared the role of fundraiser with other humans, whilst iCharibot was the only entity in charge of raising funds in our field trials. The authors do mention that donating to RoboBeggar was a lengthy process (mainly due to the use of a bank card); iCharibot attempted to avoid this, and much effort was spent on getting the robot to react to donations in a timely manner. 
To better design iCharibot, we considered other robots that work in crowded, unconstrained environments, such as Rhino, the tour-guide robot [3]. Rhino was one of the earliest robots to be able to safely move in crowded environments and showed visitors around the Deutsches Museum Bonn. The robot operated for more 47 hours, and guided more than 2000 people; perhaps more importantly the authors remark that "Rhino's ability to react to people proved to be one of the most entertaining aspects, which contributed enormously to its popularity". The same team went on to build and design Minerva [13] another tour-guide robot that operated during two weeks at the National Museum of American History in Washington D.C. The robot ran for 94 hours and performed 620 tours. Crucially, Minerva was capable of showing emotional states through a mechanical face which satisfied and amused people more than Rhino's machinelike appearance. These two robots represent early examples of the importance of human-robot interaction design when dealing with the public. Furthermore, they also proved the feasibility of robotic field trials in crowded environments.

A more recent development is the Autonomous City Explorer (ACE) [1], which went from Technische Universität München's campus to Munich's city centre by asking for directions to 38 passers-by. Similar to iCharibot, ACE received commands from passers-by via a touch screen. It covered a distance of $1.5 \mathrm{~km}$ and took 5 hours to achieve its objective.

From these studies, we identified several guidelines that were relevant for iCharibot's design: a robot should be responsive to user actions, a touchscreen can be an intuitive and reliable interface and a robot should not threaten or inconvenience passers-by.

\section{Design Considerations}

iCharibot's main role was to raise money for charitable purposes and this goal guided our design decisions. A first prototype of the robot was tested with students from our university. The lessons learnt from these preliminary tests were incorporated in iCharibot (as we describe in Sect. 3.2). $£ 48.83$ were raised from these preliminary tests.

This section details our initial choices and modifications introduced after preliminary testing in terms of both hardware and software. It also introduces the different behaviour modes which will be our primary predictor variable.

\subsection{Robot Appearance}

As can be seen in Fig. 1, iCharibot's overall appearance is human-like in size with a cylindrical body. It was built using the PeopleBot platform. We ensured iCharibot did not mislead users by appearing to be more intelligent or capable than it really was $[6]$.

To encourage interaction, a secondary Android tablet was fitted flat on the platform next to the robot's face. This interactive touch-interface allowed a user 
to play a cancer-fighting game. In addition, the robot had a three degree-offreedom servo-operated mechanical right arm (for waving) and a transparent coin box (for donation collection). We posted the Imperial Cancer Research $\operatorname{logo}^{2}$ below the coin collector surrounded by flashing blue LED strips to clearly and visibly announce iCharibot's fundraising purpose.

\subsection{Robot Software and Hardware Design}

The main computational unit for the system was a laptop PC mounted on the back of the iCharibot (on-board Robot PC in Fig. 2). This on-board PC provided physical connections via USB sockets for the Arduino microcontrollers that connected to the right arm and the coin detector. A second laptop was used to remotely-control the Onboard Robot PC (Remote $\mathrm{PC}$ in Fig. 2).

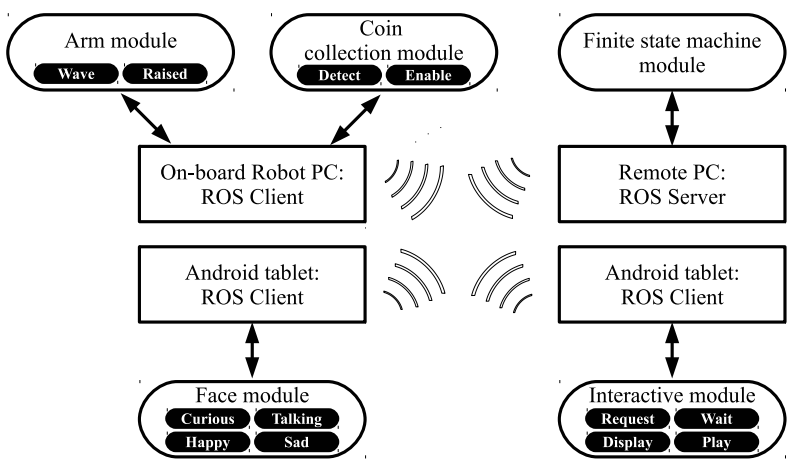

Fig. 2. iCharibot's modular software architecture. Rectangles correspond to hardware, while clear round-edged boxes represent modules. Black boxes denote the possible module states.

Figure 2 illustrates the iCharibot software architecture, developed using the Robot Operating System (ROS) [11]. In what follows, we describe each of these ROS modules.

Arm module: our three-degree-of-freedom robot arm was based on a simple model of an anthropomorphic arm. The arm servos responded to control signals from an Arduino microcontroller which in turn received commands from ROS. The arm supported two states: Wave and Raised. The former was meant to attract attention. Originally, during preliminary trials, we used high-frequency waving (at $1 \mathrm{~Hz}$ ) to make the robot appear enthusiastic but this was slowed to $0.5 \mathrm{~Hz}$ after initial tests revealed that it made iCharibot appear aggressive and prevented potential donors from approaching.

Face module: iCharibot's face, which was displayed on the Android tablet, was graphically designed to evoke emotional connections between the robot and potential donors (following $[2,15]$ ). At the same time, we avoided showing a completely human face that may induce uneasy feelings (the uncanny-valley effect [10]). There were four different facial states: Happy, Sad, Talking and $\mathrm{Cu}$ rious, with switching between states controlled by a native Android application using the rosjava library.

Coin collection module: from preliminary tests, we discovered that the robot was expected to respond in a timely manner to donations; iCharibot ini-

\footnotetext{
${ }^{2}$ Permission was granted to use Imperial Cancer Research's brand.
} 
tially used a coin sorter but we observed that this caused a donation-detection latency that led to the robot thanking the donor after she had left. This further caused confusion to the next donor. Subsequently, we replaced the coin-sorter by a flat metal receptacle attached to a microswitch which was fitted underneath the coin collector to detect coins. This allowed for immediate detection of donations, triggering the appropriate robot action.

Interactive module: This module ran on the secondary Android tablet and featured a cancer-fighting game which had been approved by Imperial Cancer Research. To play the game, users tapped their fingers on regions on the screens to "kill" cancer tumours. The game was won when all the tumours were removed.

Voice module: iCharibot synthesised audio sentences using the Festival [4] text-to-speech engine. During preliminary tests, sentences such as "Hello, how are you?" proved ineffective at attracting people's attention. As such, we redesigned iCharibot's speech to prompt users to take action (eg. "Would you like to donate for Imperial Cancer Research?"). A synthetic robotic voice was used (instead of a pre-recorded human voice) to allow donors to quickly understand they were interacting with a robot, without raising user expectations [6].

Human-detection module: iCharibot was initially programmed to detect passers-by using PeopleBot's sonars. However, this detection mechanism did not work reliably during preliminary tests, which made the robot's behaviour unpredictable. To preserve the robot interactivity aspects, we decided to manually send a human detected signal to iCharibot from our remote PC when a person approached the robot. Apart from this, iCharibot was autonomous with its actions governed by the finite state machine module.

Finite State Machine module: iCharibot's overall control algorithm was implemented using a finite state machine. The possible states of the robot organised into three distinct behaviour modes, which will be discussed next.

\subsection{Behaviour Modes}

The actions and abilities available to iCharibot during a given trial are determined by the behaviour mode. Importantly, behaviour mode is our main variable to design for greater engagement duration and donations. We devised the following modes (in order of increasing sophistication):

Baseline behaviour iCharibot stands still and ignores passers-by.

Salient behaviour iCharibot keeps waving its arm to ask for a donation but does not respond to the donors in any way when such donation is made. The tablet game is inactive in this mode.

Interactive behaviour iCharibot attempts to interact with potential donors by putting its arm down when a human approaches and asking her to play the cancer-fighting game. The game is designed to pause and iCharibot then asks for a donation. If a donation is received during the next 200 seconds, the robot will thank the donor, otherwise the robot will display an animated sad face. Notice that interactive behaviour is a superset of salient behaviour. 


\section{Experimental Procedure}

In human philanthropy studies, there is evidence that active solicitation attracts more donations than passive solicitation [9]. Might this also be true for robots? Given that saliency and interactivity imply more active solicitations, we formulated the following hypotheses:

$\mathbf{H}_{\mathbf{1}}$ A user's engagement duration $(\tau)$ with iCharibot is longer in interactive mode than in salient mode.

$\mathbf{H}_{2}$ A user's engagement duration $(\tau)$ with iCharibot is longer in salient mode than in baseline mode.

$\mathbf{H}_{3}$ The total amount collected by iCharibot is higher in interactive mode than in salient mode.

$\mathbf{H}_{4}$ The total amount collected by iCharibot is higher in salient mode than in baseline mode.

\subsection{Interaction Types}

Both $\mathbf{H}_{\mathbf{1}}$ and $\mathbf{H}_{\mathbf{2}}$ require a strict definition of what does it mean for a user to be engaged. The following categorisation of interactions between passers-by and the robot provides such definition as well as two other categories to avoid ambiguity during the field trials.

Attracted The user made a definite stop and gazed at the robot.

Engaged The user made a physical contact with iCharibot, either by touching parts of its body, arm or face; by playing the cancer-fighting game; or by making a donation. With this type of interaction we also recorded the engagement duration $(\tau)$.

Ignored If a user was neither attracted nor engaged, then we classified the interaction as ignored (even if the user did take notice of the robot).

\subsection{Engagement Duration}

In our preliminary tests, we noticed that the robot was frequently approached by groups; this made keeping track of individual engagements more complicated. We further observed that each individual in a group generally remained interested throughout the time the group was engaged with the robot; either by continuing to gaze at the robot, moving around to look its different parts or talking about it with other group members. Moreover, since iCharibot is capable of multiple engagements, individuals sometimes played the cancer-fighting game together, touched the face or arms of the robot or donated coins simultaneously. To properly account for these cases, we defined the engagement duration (denoted as $\tau$ ) as the difference between the time a user became engaged and either the time she left (if she was alone) or the whole group left (if she approached iCharibot with a group). 


\subsection{Field Trial Data Collection}

Nine field trials were carried out during February 2012 at three locations along Exhibition Road in London, easily identified by the respective landmarks: outside the Science Museum, outside the Natural History Museum and close to South Kensington station. To even out any bias, each trial was carried out at a different time of the day (between $2 \mathrm{pm}$ and $6 \mathrm{pm}$ ), with a different robot behaviour mode and at a different location.

Several pieces of information were recorded during the field trials. The most important was the interaction type of all passers-by that came close of iCharibot. For those participants who were categorised as engaged, the engagement duration $(\tau)$ was also logged. The high amount of foot traffic meant that no user surveys could be taken. Similarly, in order to ensure a swift donation process, no individual donations were recorded; note that even with a fast coin detector, individual donation tracking remains challenging as people donated concurrently. Instead of individual donations, we recorded the total funds raised per trial.

As the robot is almost fully autonomous, the duties of the researchers during experiments were limited to ensuring a safe operation and classification of interactions between passers-by and the robot. Consequently, the researchers stayed a few metres away from the robot. To aid with classification an Android application was developed which allowed for quick, convenient and concurrent categorisation of interactions. Thus a researcher only had to determine the type of interaction when a passer-by approached the robot. If the type of interaction was classified as engaged then the end of the interaction would also be logged (as per the definition of $\tau$-duration introduced in Sect. 4.2). The classification task was always carried out by the same researcher for the sake of consistency.

\section{Empirical Results, Analysis and Discussion}

Throughout our nine 40-minute trials, we recorded a total of 9884 people who came within the iCharibot's vicinity, of which 679 people were attracted to the robot and 386 people were engaged with it.

Given our definitions of engagement duration $(\tau)$ and behaviour modes, we found the median $\tau$-durations for the baseline, salient and interactive behaviours to be 18.96, 20.69 and 47.76 seconds respectively (boxplot shown in Fig. 3). To test our hypotheses, we first performed the non-parametric Kruskal-Wallis test and found the medians to be statistically different $\left(p \approx 4.9 \times 10^{-4}\right)$. A further test on pairs using the Mann-Whitney U-test showed statistical difference between the baseline and interactive modes $\left(p \approx 1.7 \times 10^{-3}\right)$, as well as the salient and the interactive modes $\left(p \approx 1.0 \times 10^{-3}\right)$. However, the test did not show statistical difference between the baseline and salient behaviours. Looking closer at the data, the minimum and maximum $\tau$-durations, discarding outliers, were $(1.00,64.74)$ for baseline mode; $(0.56,119.84)$ for salient mode; and $(0.86,219.29)$ for interactive mode. These findings are in favour of hypothesis $\mathbf{H}_{\mathbf{1}}$, but do not support hypothesis $\mathbf{H}_{\mathbf{2}}$; that is, interactivity leads to longer en- 


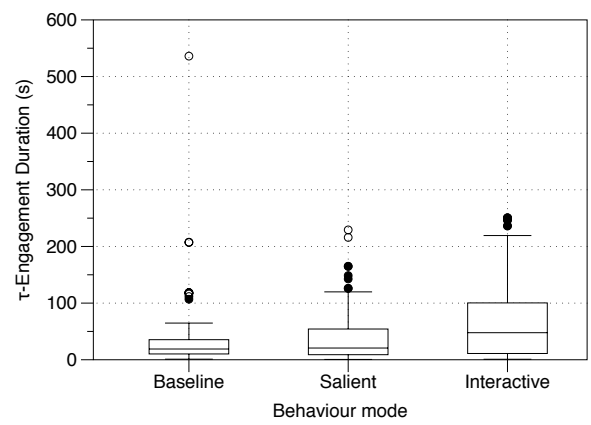

Fig. 3. Observed interaction durations (in seconds) for each behaviour mode. As hypothesised, interactivity led to an increase in the $\tau$-duration. Results shown are statistically significant with $p<0.01$.

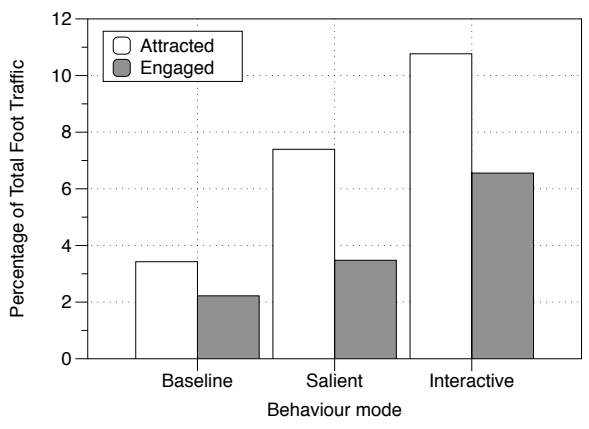

Fig. 4. Classification of interactions by percentage over total foot traffic for each behaviour mode. Note data from different locations has been collated.

gagement duration, whereas saliency alone does not appear to be sufficient for longer engagements.

Figure 4 shows a comparison of the interaction types across the three behaviour modes of iCharibot as a bar graph. The total number of people who ignored, were attracted to and engaged with iCharibot were tallied and expressed as the percentage of the total foot traffic throughout the experiment period. The percentage of passers-by who ignored the robot consistently fell from $94.4 \%$ to $89.1 \%$ to $82.6 \%$, making a step decrease of about $6 \%$ each. The percentage of passers-by who were attracted to iCharibot rose from $3.4 \%$ to $7.4 \%$ to $10.8 \%$ and of those who were engaged with iCharibot from $2.2 \%$ to $3.5 \%$ to $6.6 \%$. Thus, as iCharibot's behaviour became more sophisticated, fewer passers-by ignored it and more of them were attracted to or became engaged with the robot.

Figure 5 shows the average amount of funds collected per trial (in pence sterling) over the total foot traffic per trial - note that this amount is not representative of the amount of money donated by any single person. As can be observed from the figure, the data suggests that the funds raised increased with more sophisticated iCharibot behaviours. The average donation per person was $0.39 \mathrm{p}$ for baseline mode, $0.84 \mathrm{p}$ for salient mode and $1.19 \mathrm{p}$ for interactive mode;

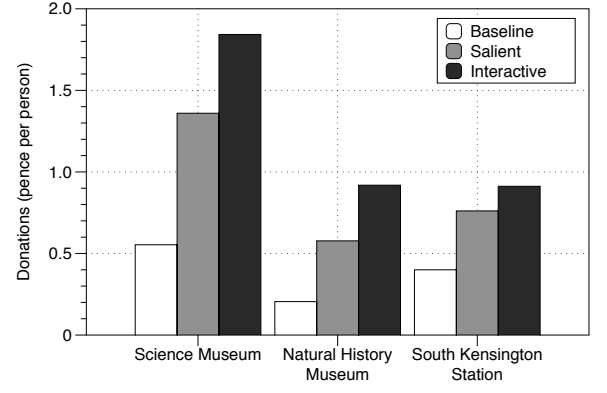

Fig. 5. Average donation amount per person, calculated as the money collected (in pence) over the total foot traffic per trial. amounts are pence sterling and were calculated dividing the funds raised over the total flow. This represents an increase in average donations of around $0.4 \mathrm{p}$ as the sophistication of the robot grows, suggestive of $\mathbf{H}_{\mathbf{3}}$ and $\mathbf{H}_{\mathbf{4}}$. However, without individual donations, we cannot compute the statistical validity of these findings. 
In addition, the average donation per engaged person was 17.35p for baseline mode, $24.21 \mathrm{p}$ for salient mode, and $18.09 \mathrm{p}$ for interactive mode; these values were calculated dividing the funds raised over the number of engaged people ${ }^{3}$. We find surprising that the interactive mode yields a lower average than the salient mode and speculate that it may be due to interactive mode getting a higher portion of its contribution from groups where only one person donated for everyone. Irrespectively, this fact deserves more attention and will be the subject of future research.

In summary, our results suggest that saliency is only enough to attract more passers-by but not to make them interact with the robot for longer. In order to achieve longer human-robot interactions, a more sophisticated behaviour was needed. In addition, we also observed promising evidence that a more sophisticated robot behaviour leads to a greater donation amount.

\section{Conclusion and Further Work}

In this article, we presented the design and realisation of iCharibot, a fundraising robot. We worked towards a robot that appeared friendly with cartoon-like facial expressions and ensured that it was responsive to donations. During experiments in a very busy area of London, 386 people engaged with iCharibot, and $£ 75.94$ sterling was raised for Imperial Cancer Research (plus an extra $£ 48.83$ in preliminary tests).

Overall, we found that increasing behaviour sophistication appealed to people, causing them to spend more time with iCharibot. Our results confirmed several intuitive notions: robot interactivity leads to a higher level of engagement while saliency alone does not (under the assumptions presented in Sect. 4.2). Moreover our data suggests that interactivity also leads to a higher amount of donations - we look forward to more tests with iCharibot, where individual donation amounts are recorded, to conclusively show this.

This work opens up several avenues for future research. From a technical standpoint, iCharibot can be improved in several ways. For example, sonars proved too unreliable for automatically detecting users and we are currently investigating the use of a Kinect sensor as a potential solution. From a HRI standpoint, interacting with iCharibot could be richer and more fluid. For example, communicating through a touch interface is effective and reliable but not as intuitive as speech. From an experimental perspective, we used a conservative classification; during the field-trials, many people glanced and smiled at the robot but were classified as ignored. Future work would go into refining these classifications in order to better capture the range of interactions that occurred. Another question left to be explored is whether interactivity is actually cost effective. The data suggests that interactivity yields higher donations at the expense of higher engagement time. This expense might not be without benefits since we have also observed a "group effect", where passers-by that walked in

\footnotetext{
${ }^{3}$ Note that, as per the definitions in 4.1 , everyone who donates is classified as engaged, but not every engaged person donated.
} 
groups were more likely to approach the robot $([12,14]$ report similar findings). Finding principled methods for balancing the multitude of factors that influence how engaging a robot is for a certain task remains a challenging and rewarding task that deserves further consideration.

\section{References}

1. Bauer, A., Klasing, K., Lidoris, G., Mühlbauer, Q., Rohrmüller, F., Sosnowski, S., Xu, T., Kühnlenz, K., Wollherr, D., Buss, M.: The autonomous city explorer: Towards natural human-robot interaction in urban environments. Intl $\mathrm{J}$ of Social Robot. 1(2), 127-140 (2009)

2. Breazeal, C.: Emotion and sociable humanoid robots. Intl J Human-Computer Stud. 59(1-2), 119-155 (2003)

3. Burgard, W., Cremers, A., Fox, D., Hähnel, D., Lakemeyer, G., Schulz, D., Steiner, W., Thrun, S.: Experiences with an interactive museum tour-guide robot. Artif. Intell. 114(1-2), 3-55 (1999)

4. Clark, R.A., Richmond, K., King, S.: Festival 2 - Build Your Own General Purpose Unit Selection Speech Synthesiser. In: Proc of ISCA Speech Synthesis Workshop. pp. $173-178$ (2004)

5. Dobbs, J., Jochum, V., Wilding, K., Lipscomb, L., Smith, M., Harrison, R.: UK Giving 2011: An overview of charitable giving in the UK (2011)

6. Goetz, J., Kiesler, S., Powers, A.: Matching robot appearance and behavior to tasks to improve human-robot cooperation. In: Proc of IEEE RO-MAN. pp. 55-60 (2003)

7. Kim, M.S., Cha, B.K., Park, D.M., Lee, S.M., Kwak, S., Lee, M.K.: Dona : Urban Donation Motivating Robot. In: Proc of ACM/IEEE HRI. pp. 159-160 (2010)

8. La Russa, G., Sutinen, E., Cronje, J.C.: When a robot turns into a totem: The RoboBeggar case. In: Soomro, S. (ed.) E-learning Experiences and Future, chap. 17, pp. 327-344. InTech (2010)

9. Lindskold, S., Forte, R.A., Haake, C.S., Schmidt, E.K.: The Effects of Directness of Face-to-Face Requests and Sex of Solicitor on Streetcorner Donations. J of Social Psychology 101, 45-51 (1977)

10. Mori, M.: The uncanny valley. Energy 7(4), 33-35 (1970)

11. Quigley, M., Gerkey, B., Conley, K., Faust, J., Foote, T., Leibs, J., Berger, E., Wheeler, R., Ng, A.: ROS : an open-source Robot Operating System. In: Proc of the Open Source Software Workshop at ICRA (2009)

12. Shiomi, M., Sakamoto, D., Kanda, T., Ishi, C.T., Ishiguro, H., Hagita, N.: Field Trial of a Networked Robot at a Train Station. Intl J of Social Robot. 3(1), 27-40 (2010)

13. Thrun, S., Bennewitz, M., Burgard, W., Cremers, A., Dellaert, F., Fox, D., Hahnel, D., Rosenberg, C., Roy, N., Schulte, J., Others: MINERVA: A second-generation museum tour-guide robot. In: Proc of IEEE ICRA. pp. 1999-2005 (1999)

14. Weiss, A., Bernhaupt, R., Tscheligi, M., Wollherr, D., Kuhnlenz, K., Buss, M.: A methodological variation for acceptance evaluation of Human-Robot Interaction in public places. In: Proc of IEEE RO-MAN. pp. 713-718 (2008)

15. Yuasa, M., Saito, K., Mukawa, N.: Brain activity associated with graphic emoticons. the effect of abstract faces in communication over a computer network. Electrical Engineering in Japan 177(3), 36-45 (2011) 